Gefässchirurgie 2009 · 14:77-78 DOI 10.1007/s00772-009-0673-5

Online publiziert: 21. März 2009

(c) Springer Medizin Verlag 2009
V. Mickley ${ }^{1}$ M. Hollenbeck ${ }^{2} \cdot$ P. Haage ${ }^{3}$

${ }^{1}$ Fachbereich Gefäßchirurgie, Deutsche Gesellschaft für Gefäßchirurgie, Kreiskrankenhaus Rastatt

${ }^{2}$ Klinik für Nephrologie und Rheumatologie, Deutsche Gesellschaft für Nephrologie, Knappschaftskrankenhaus Bottrop

${ }^{3}$ Radiologie Region West, Deutsche Röntgengesellschaft, HELIOS Klinikum Wuppertal

\title{
Interdisziplinäre Empfehlungen deutscher Fachgesellschaften zum Gefäßzugang zur Hämodialyse
}

Vor dem Hintergrund der stetig steigenden Zahl dialysepflichtiger Patienten stellt der Umgang mit dem Gefäßzugang zur Hämodialyse für alle Beteiligten eine wachsende Herausforderung dar, insbesondere im Hinblick auf die stetig steigende Zahl älterer und gefäßkranker Dialysepatienten. Vor diesem Hintergrund haben sich in den vergangenen Jahren interessante und wertvolle Initiativen entwickelt, die im Folgenden kurz dargestellt werden sollen. Der stets interdisziplinäre Ansatz dieser Aktivitäten macht sie besonders attraktiv. Nur durch die enge Zusammenarbeit der mit Gefäßzugängen befassten Berufsgruppen (Nephrologie und nephrologische Pflege, Gefäßchirurgie, diagnostische und interventionelle Angiologie und Radiologie) wird auch in Zukunft ein hoher Behandlungsstandard aufrechtzuerhalten sein. Gerade bei der Behandlung zugangsassoziierter Probleme und Komplikationen macht die Vielzahl der möglichen Diagnose- und Therapiestrategien die Erarbeitung von fachübergreifenden Richtlinien erforderlich, um jedem einzelnen Patienten ein optimales Behandlungskonzept anbieten zu können.

\section{Neue DGG-Leitlinie "Shuntchirurgie"}

Im September 2008 wurden die aktuellen Leitlinien der Deutschen Gesellschaft für Gefäßchirurgie (DGG) auf ihrem Jahreskongress publiziert und stehen seither auf der Website der DGG (http://www.gefaesschirurgie.de) zum Download bereit. Seit Kurzem sind sie auch von der Arbeitsgemeinschaft der Wissenschaftlichen Medizinischen Fachgesellschaften (AWMF) in ihren Leitlinienkatalog (http://leitlinien. net) aufgenommen worden. Eine Publikation in Buchform beim Deutschen Ärzteverlag ist in Vorbereitung.

Im Vergleich zu ihrer Vorgängerversion wurden die Leitlinien der DGG nicht nur aktualisiert, sondern auch um einige wichtige Kapitel erweitert. Unter anderem wurde der zunehmenden Bedeutung der Shuntchirurgie im Alltag des Gefäßchirurgen Rechnung getragen und ihr ein eigenes Kapitel gewidmet (http:// www.gefaesschirurgie.de/upload/dl/Leitlinien2008/Shuntchirurgie.pdf). Bei seiner Erarbeitung hat die Leitlinienkommission der DGG besonderen Wert auf die Einbindung der übrigen besonders am Dialysezugang interessierten Fachgesellschaften, der Deutschen Gesellschaft für Nephrologie (DGN), der Deutschen
Röntgengesellschaft (DRG) und der Deutschen Gesellschaft für Angiologie (DGA) gelegt. Neben Kapiteln zur präoperativen Diagnostik und zu technischen Aspekten der Erstanlage von arteriovenösen Fisteln und Prothesenshunts finden sich in dieser Leitlinie ausführliche Darstellungen der chirurgischen und interventionellen Therapie des gesamten Spektrums der möglichen fistel- und prothesenassoziierten Komplikationen, von peripheren und zentralvenösen Stenosen über Aneurysma und Infektion bis hin zur hochflussassoziierten Herzinsuffizienz und zugangsassoziierten Ischämiesyndromen.

\section{Die neuen „Interdisziplinären Empfehlungen"}

Parallel zur Erarbeitung der Gefäßchirurgischen Leitlinien konstituierte sich auf Initiative der Deutschen Arbeitsgemeinschaft für klinische Nephrologie im Februar 2008 die GHIA (Gefäßzugänge für die Hämodialyse: Interdisziplinäre Arbeitsgruppe) Neben den Autoren dieses Beitrags wurden von den Vorständen der bereits genannten Fachgesellschaften weitere Mitglieder in diese Arbeitsgruppe entsandt: Jan Brunkwall (Köln) und Harald Daum (Hamburg) für die DGG, Jürgen Ranft (Bottrop) für die DGA, Ralf 
Schindler (Berlin) und Peter Thon (Bad Hersfeld) für die DGN sowie Dierk Vorwerk (Ingolstadt) für die DRG.

Erklärtes Ziel der GHIA ist die Stärkung der interdiszipinären Zusammenarbeit zum Zwecke der landesweiten Verbesserung der Versorgung unserer Hämodialysepatienten im Hinblick auf zugangsasssoziierte Fragen und Probleme. Das erste Projekt der GHIA stellte die interdisziplinäre Erarbeitung von Empfehlungen zum Umgang mit Gefäßzugängen zur Hämodialyse dar. Diese Empfehlungen werden nach zwischenzeitlich erfolgter Genehmigung durch die Vorstände der beteiligten Fachgesellschaften nun im Märzheft des „Nephrologen“ publiziert (Hollenbeck $\mathrm{M}$ et al. (2009) Interdisziplinäre Empfehlungen deutscher Fachgesellschaften zum Gefäßzugang zur Hämodialyse. Nephrologe 4:158-176). Zeitgleich wird das Dokument auf der Homepage der "Gefässchirurgie“ zum kostenlosen Download zur Verfügung gestellt (www.gefaesschirurgie.springer.de).

Die bereits 2007 erschienen European Best Practice Guidelines (Tordoir J et al. (2007) EBPG on vascular access. Nephrol Dial Transplant 22 (Suppl 2): ii88-ii117) dienten bei der Erstellung der deutschen Empfehlungen als Ausgangspunkt. Sie wurden von der GHIA überarbeitet, durch Sichtung aktueller Literatur auf den neuesten Stand gebracht, teilweise neu strukturiert und um einige Kapitel ergänzt. Somit ist die GHIA nun erstmals in der Lage, eine den aktuellen Bedürfnissen und Gegebenheiten in unserem Land angepasste Sammlung interdisziplinärer Empfehlungen zum Dialysezugang zu präsentieren, die in der Zusammenarbeit aller beteiligten Fachgesellschaften entstanden ist und von deren Zustimmung getragen wird. In diesem Zusammenhang besonders erwähnenswert ist die Tatsache, dass auf Anregung des Vorstands der DGG unsere Empfehlungen als Zweitabdruck zusammen mit den Leitlinien der DGG in Buchform erscheinen werden. Durch die breit gefächerte Publikation (in Zeitschrift, Buch und Internet) wird es möglich, die Empfehlungen dem interessierten Publikum leicht zugänglich zur Verfügung zu stellen.

Der Leser der „Interdisziplinären Empfehlungen“ wird feststellen, dass sie nicht in Konkurrenz zu den „Leitlinien Shuntchirurgie" treten, sondern diese in wesentlichen nichtchirurgischen Bereichen erweitern und ergänzen. Großer Raum wurde der Diagnostik vor und der Nachsorge nach Shuntanlage eingeräumt, und natürlich fehlen auch Kapitel zur Indikation und Implantation von Dialysekathetern sowie zur Therapie katheterassoziierter Komplikationen nicht. Alle Kapitel werden jeweils von kurzen, evidenzbasierten Grundsatzempfehlungen eingeleitet, die im Folgenden dann anhand einer ausführlichen Wertung der aktuellen Literatur (insgesamt 290 Zitate) erläutert und begründet werden.

Nephrologisch-pflegerische Gesichtspunkte wurden bei der Erarbeitung unserer Empfehlungen bewusst weitgehend ausgespart. Wiederum parallel zu den Aktivitäten der medizinischen Fachgesellschaften hat nämlich im Juli 2007 der Fachverband nephrologischer Berufsgruppen (fnb) eine interdisziplinäre und interprofessionelle Arbeitsgruppe ins Leben gerufen, die sich mit eben diesen Themen auseinandersetzt. Die GHIA hat diese Arbeiten begleitet. Die Publikation der pflegerischen Empfehlungen ist für den Herbst 2009 geplant.

\section{Weitere GHIA-Aktivitäten}

Um bei der teilweise babylonisch anmutenden Verwirrung der Begrifflichkeiten im Zusammenhang mit Gefäßzugängen mehr Klarheit zu schaffen, haben wir gemeinsam mit den Pflegeverbänden einen Vorschlag zur deutschen Nomenklatur des Dialysezugangs erstellt, den wir unter der Adresse www.Der Nephrologe.de zur Diskussion stellen und im Herbst 2009 festschreiben wollen. Dies erscheint unabdingbar im Hinblick auf die eigentlichen zukünftigen Zielsetzungen der GHIA: die Intensivierung der Zusammenarbeit der beteiligten Fachgesellschaften zur Förderung der Weiterbildung und Forschung auf dem Gebiet des Gefäßzugangs zur Hämodialyse, die Evaluation der aktuellen Versorgungssituation und die Entwicklung von Qualitätskriterien. Fernziel ist die Formulierung von strukturellen und qualitativen Anforderungen an Shuntschwerpunktzentren sowie - wiederum in Zusammenarbeit mit den beteiligten Fachgesellschaften - deren Zertifizierung.

\section{Korrespondenzadresse \\ Dr. V. Mickley \\ Fachbereich Gefäßchirurgie, \\ Kreiskrankenhaus Rastatt \\ Engelstr. 39, 76437 Rastatt \\ v.mickley@klinikum-mittelbaden.de}

Danksagung. Die Autoren danken im Namen der GHIA allen beteiligten Fachgesellschaften und ihren Vorständen für deren Unterstützung unserer Arbeiten. Dem Springer-Verlag gilt unser besonderer Dank nicht nur wegen des Abdrucks unserer Empfehlungen in "Der Nephrologe", sondern vor allem auch wegen der Einrichtung des kostenfreien Möglichkeit zum Download und der unbürokratischen Abtretung der Rechte zum Zweitabdruck in Buchform.

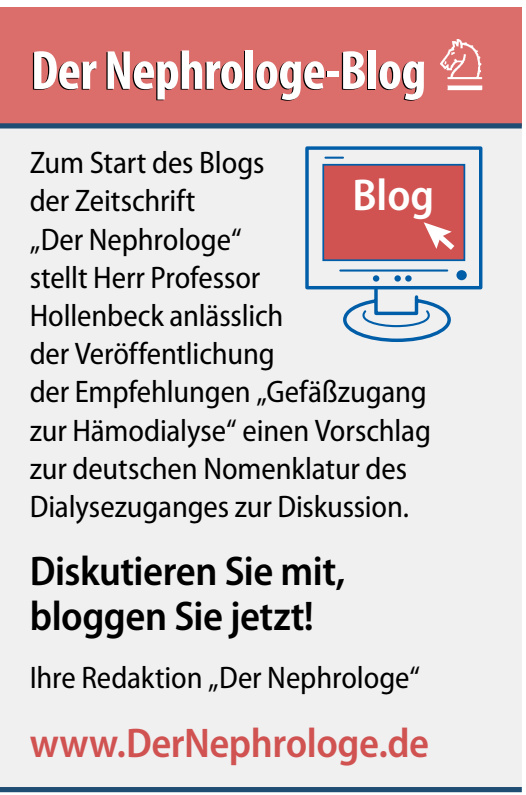

